\title{
The basic helix-loop-helix transcription factor TabHLH1 increases chlorogenic acid and luteolin biosynthesis in Taraxacum antungense Kitag
}

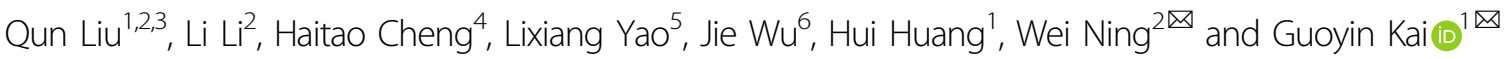

\begin{abstract}
Polyphenols are the main active components of the anti-inflammatory compounds in dandelion, and chlorogenic acid (CGA) is one of the primary polyphenols. However, the molecular mechanism underlying the transcriptional regulation of CGA biosynthesis remains unclear. Hydroxycinnamoyl-CoA:quinate hydroxycinnamoyl transferase (HQT2) is the last rate-limiting enzyme in chlorogenic acid biosynthesis in Taraxacum antungense. Therefore, using the TaHQT2 gene promoter as a probe, a yeast one-hybrid library was performed, and a basic helix-loop-helix (bHLH) transcription factor, TabHLH1, was identified that shared substantial homology with Gynura bicolor DC bHLH1. The TabHLH1 transcript was highly induced by salt stress, and the TabHLH1 protein was localized in the nucleus. CGA and luteolin concentrations in TabHLH1-overexpression transgenic lines were significantly higher than those in the wild type, while CGA and luteolin concentrations in TabHLH1-RNA interference (RNAi) transgenic lines were significantly lower. Quantitative realtime polymerase chain reaction demonstrated that overexpression and RNAi of TabHLH1 in T. antungense significantly affected CGA and luteolin concentrations by upregulating or downregulating CGA and luteolin biosynthesis pathway genes, especially TaHQT2, 4-coumarate-CoA ligase (Ta4CL), chalcone isomerase ( $T a C H)$, and flavonoid-3'-hydroxylase (TaF3'H). Dual-luciferase, yeast one-hybrid, and electrophoretic mobility shift assays indicated that TabHLH1 directly bound to the bHLH-binding motifs of proTaHQT2 and proTa4CL. This study suggests that TabHLH1 participates in the regulatory network of CGA and luteolin biosynthesis in T. antungense and might be useful for metabolic engineering to promote plant polyphenol biosynthesis.
\end{abstract}

\section{Introduction}

Dandelions (Taraxacum spp.) have been used as medicinal herbs and functional foods for several centuries ${ }^{1,2}$. The increasing demand for dandelion products, such as tea, wine, syrup, and coffee, has promoted the industrialization of dandelion production ${ }^{3}$. The total phenolic compound concentrations in different tissues of Taraxacum mongolicum

\footnotetext{
Correspondence: Wei Ning (1025265208@qq.com) or

Guoyin Kai (guoyinkai@yahoo.com)

'Laboratory for Core Technology of TCM Quality Improvement and Transformation, College of Pharmacy, School of Pharmaceutical Sciences, The Third Affiliated Hospital, Zhejiang Chinese Medical University, Hangzhou, Zhejiang 310053, PR China

${ }^{2}$ College of Horticulture, Shenyang Agricultural University, Shenyang 110866, China Full list of author information is available at the end of the article

These authors contributed equally: Qun Liu, Li Li
}

ranged from 37.12 to $68.89 \mathrm{mg} \mathrm{GAE} / \mathrm{g}^{4-7}$. However, these levels in Taraxacum antungense have not been tested in previous studies. Polyphenolic compounds, including phenolic acids, flavonoids, and anthocyanins, have many biological activities ${ }^{8-10}$. In Taraxacum antungense Kitag, chlorogenic acid (CGA) and caffeic acid (CA) have antioxidative benefits, being hepatoprotective and having diuretic activities $^{11-13}$; rutin and luteolin are used to treat several diseases, such as Parkinson's disease, severe acute respiratory syndrome, hepatitis, and cancer ${ }^{14-16}$. However, the concentration of these functional active constituents in $T$. antungense is lower than that in other medicinal plants, such as Lonicera japonica and Eucommia ulmoides, which restricts dandelion industrialization ${ }^{14}$. Bioengineering strategies could potentially increase the polyphenolic compounds

\section{(c) The Author(s) 2021}

(c) (i) Open Access This article is licensed under a Creative Commons Attribution 4.0 International License, which permits use, sharing, adaptation, distribution and reproduction in any medium or format, as long as you give appropriate credit to the original author(s) and the source, provide a link to the Creative Commons license, and indicate if changes were made. The images or other third party material in this article are included in the article's Creative Commons license, unless indicated otherwise in a credit line to the material. If material is not included in the article's Creative Commons license and your intended use is not permitted by statutory regulation or exceeds the permitted use, you will need to obtain permission directly from the copyright holder. To view a copy of this license, visit http://creativecommons.org/licenses/by/4.0/. 


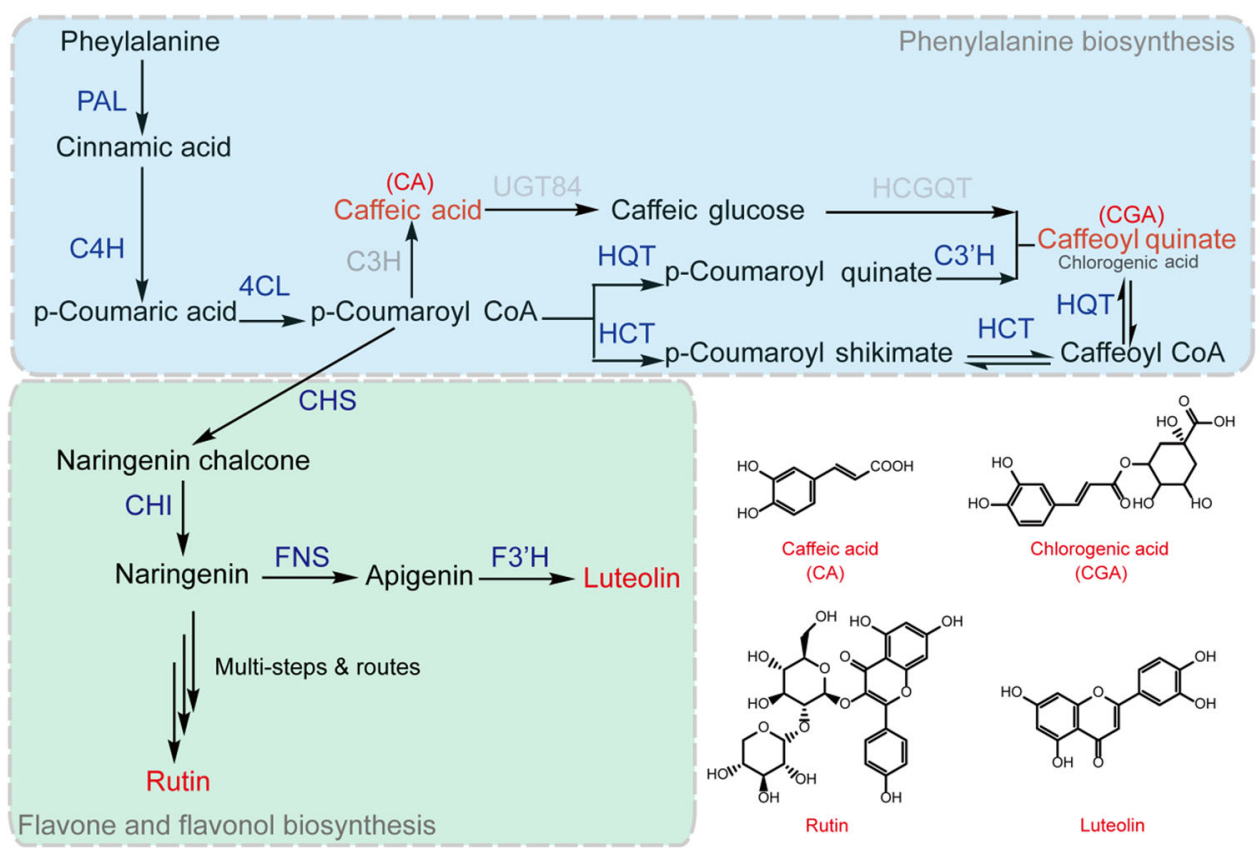

Fig. 1 Biosynthetic pathway of the main polyphenols (caffeic acid, chlorogenic acid, rutin, and luteolin) in Taraxacum antungense. Phenylalanine ammonia-lyase (PAL); cinnamate 4-hydroxylase (C4H); 4-coumarate-CoA ligase (4CL); Coumarate 3-hydroxylase (C3H); 5-O-(4coumaroyl)-D-quinate 3'-monooxygenase (C3'H); hydroxycinnamoyl-coenzyme (Co)A shikimate/quinate hydroxycinnamoyl transferase (HCT); hydroxycinnamoyl-CoA quinate hydroxycinnamoyl transferase (HQT); caffeoylshikimate esterase (CSE); UDP-glucosyltransferase (UGT84); Chalcone synthase (CHS); flavonoid synthase (FNS); Chalcone isomerase (CHI); and hydroxycinnamoyl D-glucose quinate hydroxycinnamoyl transferase (HCGQT). Three arrows indicate multi-steps \& routes. Blue indicates what has been reported in this article, and gray indicates information from other plants

in Taraxacum; however, a better understanding of the polyphenolic compound biosynthesis pathway is required.

Since the 1990s, the polyphenol biosynthesis pathway has been reported in several medicinal model plants, such as Salvia miltiorrhiza, L. japonica, and Dendranthema morifolium $^{17,18}$. In the first three steps of polyphenol biosynthesis, the key enzymes are phenylalanine ammonia-lyase (PAL), 4-coumarate-CoA ligase (4CL), and cinnamate 4-hydroxylase $(\mathrm{C} 4 \mathrm{H})$, which catalyze the synthesis of p-coumaroyl-CoA from phenylalanine ${ }^{19}$. CA is directly catalytically synthesized by coumarate 3-hydroxylase from p-coumaroyl-CoA, whereas CGA is catalytically synthesized by hydroxycinnamoyl CoA quinate hydroxycinnamoyl transferase (HQT) and hydroxycinnamoyl-CoA shiki-mate/quinate hydroxycinnamoyl transferase $(\mathrm{HCT})^{20}$. However, in T. antungense, only HQTs (TaHQT1/2) have been isolated, identified, and assessed, and TaHQT2 is the last ratelimiting enzyme in chlorogenic acid biosynthesis ${ }^{21}$. For flavonoids, p-coumaroyl-CoA is a branch and precursor compound. The first two steps in the catalytic synthesis of naringenin from p-coumaroyl-CoA are via chalcone synthase and chalcone isomerase $(\mathrm{CHS}$ and $\mathrm{CHI}$, respectively $)^{8}$. Naringenin is catalyzed by flavonoid synthase and flavonoid-3'-hydroxylase (FNS and ${ }^{\prime} 3^{\prime} \mathrm{H}$, respectively) in plants to synthesize luteolin ${ }^{8,22}$. Naringenin is also catalyzed by flavonol-3-dehydrogenase (F3H), flavonol synthetase (FLS), and UDP-glucoronosyl/UDP-glucosyl transferase (UFGT) to synthesize rutin ${ }^{23-25}$. PAL, CHS, $\mathrm{CHI}$, and $\mathrm{F}^{\prime}{ }^{\prime} \mathrm{H}$ increase CGA and luteolin concentrations in L. japonica, whereas in Fagopyrum tataricum, the rutin concentration is increased by upregulation of FLS and UFGT gene expression ${ }^{25,26}$. Identification of these polyphenol biosynthesis pathway genes will lay the foundation for further genetic engineering research in $T$. antungense (Fig. 1).

Plant polyphenolic compounds are important components acting against biotic and abiotic stresses ${ }^{21,25}$. Plant transcription factor (TF) family members, such as MYB11/12/111 and WRKY18/40/60, respond to biotic and abiotic stress to regulate the expression of downstream structural genes and ultimately promote the biosynthesis of polyphenols ${ }^{18,19,27}$. Coexpression analysis of TFs and biosynthesis pathway gene expression levels often showed a highly linear relationship ${ }^{27,28}$. Thus, TFs may be used as a tool not only to improve a plant's ability to adapt to the environment but also to increase polyphenolic compound production in plants ${ }^{21}$.

Basic helix-loop-helix (bHLH) TFs are one of the largest families that regulate the expression of key enzyme genes 
in plants ${ }^{28}$. Among these TFs, myelocytomatosis oncogene (MYC) TFs (representative bHLH TFs) often participate in secondary metabolite accumulation: AaMYC2 (Artemisia annua L) regulates artemisinin biosynthesis, NtMYC2 (Nicotiana tabacum) regulates nicotine biosynthesis, and CrMYC2 (Catharanthus roseus) regulates the expression of alkaloid biosynthesis genes that respond to methyl jasmonate (MeJA) ${ }^{29,30}$. In the medicinal model plant S. miltiorrhiza, SmMYC2a/2b regulates key enzyme genes for phenolic acid biosynthesis ${ }^{31}$. Upregulation of SmPAL by SmbHLH37 leads to increased phenolic acid accumulation ${ }^{32}$. Moreover, the MeJA-responsive SmbHLH53 TF regulates enzymatic genes involved in the salvianolic acid B biosynthesis pathway in S. miltiorrhiza $^{33}$. However, the function of bHLH TFs in $T$. antungense polyphenol biosynthesis and their regulatory models have been poorly reported ${ }^{34}$.

TFs regulate gene expression levels by combining with cis-acting elements of functional gene promoters; bHLH TFs specifically bind to E-box ${ }^{28,35}$. In T. antungense, the TaHQT2 gene promoter was obtained and was found to contain various cis-acting elements, specifically four E-boxes (CANNTG) (Supplementary Fig. S1 and Table S2). E-boxes are widely distributed in the promoter region of key enzymes in polyphenol biosynthesis ${ }^{36}$. Therefore, it was speculated that bHLH TFs bind to the TaHQT2 promoter to participate in polyphenol biosynthesis in $T$. antungense.

In this study, a T. antungense bHLH TF, TabHLH1, which was obtained through yeast one-hybrid $(\mathrm{Y} 1 \mathrm{H})$ screening, had high homology to bHLH1 from the Gerbera hybrid cultivar. Polyphenol concentration analysis and quantitative real-time polymerase chain reaction (qRT-PCR) results showed that TabHLH1 increased CGA and luteolin biosynthesis by increasing TaHQT2, Ta4CL, TaCHI, and $\mathrm{TaF}^{\prime} \mathrm{H}$ gene expression levels in $T$. antungense transgenic lines. Functional analysis of TabHLH1 suggested that it regulates the biosynthesis of CGA and luteolin, enhancing the understanding of the routes of polyphenol biosynthesis and providing a structure for future metabolic engineering of $T$. antungense.

\section{Results}

\section{Isolation and characterization of TabHLH1}

To identify bHLH TFs involved in CGA biosynthesis of T. antungense, $\mathrm{Y} 1 \mathrm{H}$ assays were applied to screen the $T$. antungense cDNA library, and the TaHQT2 promoter was used as bait. Approximately $860 \mathrm{bp}$ of the TaHQT2 promoter sequence (proTaHQT2) was cloned after two rounds of amplification, and several elements were identified, including an androgen response element, TATAbox, CAAT-box, CGTCA/TGACG-motif, estrogenresponsive element, long terminal repeat, E-box, P-box,
TGA-box, and light-responsive elements (Fig. S1 and Table S2). A Y1H cDNA library of $T$. antungense was created with a titer of approximately $5 \times 10^{7}$ colonyforming units $/ \mathrm{mL}$. PCR results showed that the length of T. antungense cDNA ranged from $200-2000$ bp (Fig. S2). A 200 bp DNA fragment containing four E-boxes (proTaHQT2-E-box-1, -2, -3, and -4) that were identified in proTaHQT2 (from -685 to -810 ) within $860 \mathrm{bp}$ of the ATG start codon was cloned. The isolated gene coding protein was able to bind to the proTaHQT2 CATGTG motif (Fig. 2). The results also showed that pMutantTaHQT2 interacted with the isolated gene coding protein.

The isolated protein acquired from the nonredundant protein database (using the Basic Local Alignment Search Tool for protein [BLASTP]) showed high homology to the Gerbera hybrid cultivar bHLH1 (GhbHLH1: 69\% identity and $78 \%$ positivity) and Gynura bicolor bHLH1 (GbbHLH: $66 \%$ identity and $76 \%$ positivity) (Fig. 3A). The full-length TabHLH1 cDNA was 1566 bp and encoded a 521-amino acid protein (accession number: MH683054) (Fig. 3B). Multiple sequence alignment identified a conserved bHLH-MYC_N domain and an HLH domain in TabHLH1 (pfam 14215 and pfam 00010) using the Pfam online tool (http://pfam.xfam.org/search/sequence). Based on these results, we deduced that TabHLH1 was a bHLH TF.

Subcellular localization showed that the yellow fluorescent protein (YFP) (control group; empty vector) was distributed uniformly throughout the cell, whereas TabHLH1-YFP fluorescence (treatment group; TabHLH1-YFP vector) was observed in the nucleus. This was confirmed using the positive control nuclear stain 4',6-diamidino-2-phenylindole (DAPI) (Fig. 3C).

\section{Biochemical analyses of the main polyphenol compounds in T. antungense and expression profiling of TabHLH1}

The total phenolic concentrations of $T$. antungense in different tissue samples ranged from $32.37-66.23 \mathrm{mg}$ $\mathrm{GAE} / \mathrm{g}$, and the highest concentrations of total phenolics were found in flowers, followed by leaves, roots, and stems (Fig. 4A, B). The four phenolic acids (CGA, CA, rutin, luteolin) in different tissues of $T$. antungense showed significant differences. CGA concentrations were highest in the roots, followed by flowers, stems, and leaves; CA concentrations were highest in the flowers, followed by leaves and flowers; rutin concentrations were highest in roots and flowers, followed by leaves and stems; luteolin concentrations were highest in leaves, followed by flowers and roots, while $\mathrm{CA}$ and luteolin were not detected in stems (Fig. 4C).

To study the correlation between key polyphenol biosynthesis enzyme genes and TabHLH1 TF in different tissues, one-year-old wild flowering $T$. antungense was used for expression analysis by qRT-PCR. The results 

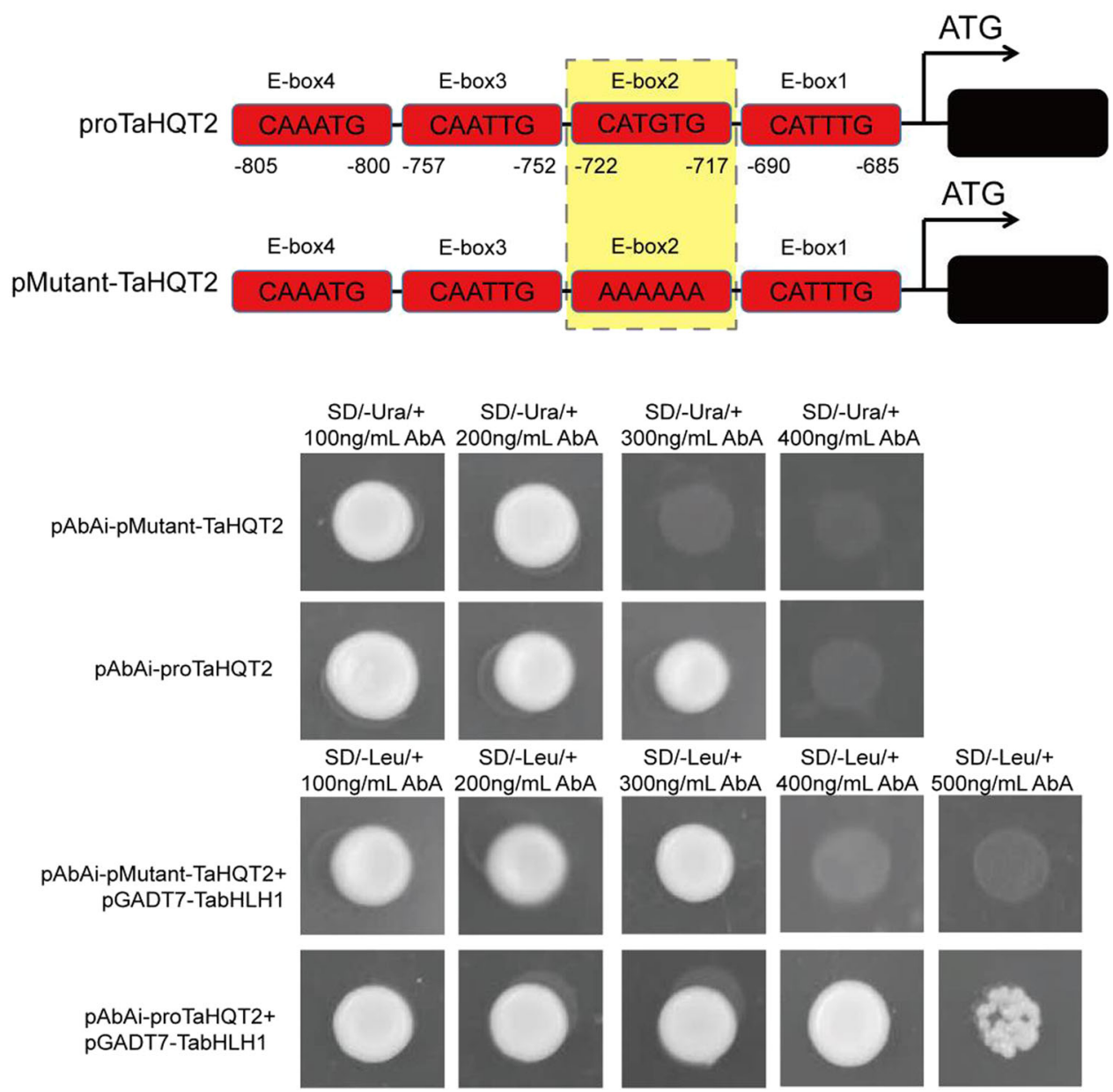

Fig. 2 Y1H assay. pAbAi-E-box grew normally on $300 \mathrm{ng} / \mathrm{mL}$ aureobasidin A (AbA); however, pAbAi-pMutant-E-box was inhibited by $300 \mathrm{ng} / \mathrm{mL}$ $\mathrm{AbA}$. Therefore, $400 \mathrm{ng} / \mathrm{mL}$ was the minimum concentration of AbA used to examine the interaction between TabHLH1 and the E-boxes. We used the pAbAi-E-box and pAbAi-pMutant-E-box to transform $\mathrm{Y} 1 \mathrm{H}$ gold yeast and then made new $\mathrm{Y} 1 \mathrm{H}$ competent cells. Subsequently, the pGADT7TabHLH1 recombinant vector was transformed into new $\mathrm{Y} 1 \mathrm{H}$ competent cells. The cotransformed pAbAi-E-box + pGADT7-TabHLH1 grew on 400-500 ng/mL AbA; however, pAbAi-pMutant-E-box + pGADT7-TabHLH1 was unable to grow and needed a higher concentration of AbA. The difference between the pAbAi-E-box and pAbAi-pMutant-E-box was that the E-box pAbAi-E-box contained CAAATG, CATTTG, CATGTG, and CAATTG motifs. In the pAbAi-pMutant-E-box, AAAAAA was used instead of the CATGTG motif

showed that HQT1 was highly expressed in roots; CHS, $\mathrm{CHI}, 4 \mathrm{CL}, \mathrm{FNS}$, and F3' $\mathrm{H}$ were highly expressed in flowers followed by leaves; HCT, PAL, HQT2, and C4H were highly expressed in leaves followed by flowers. For TabHLH1, the expression level was highest in leaves, followed by flowers, roots, and stems (Fig. 4D).

To study the effects of various biotic and abiotic stresses on the expression level of TabHLH1, NaCl, MeJA, salicylic acid (SA), abscisic acid (ABA), ethephon (ET), and gibberellic acid (GA3) treatments were performed at different time points in $T$. antungense leaves, and then qRTPCR was used for expression analysis. Under $\mathrm{NaCl}$ treatment $(500 \mathrm{ng} / \mathrm{mL})$, TabHLH1 expression increased more than 40 -fold in $3 \mathrm{~h}$, indicating that TabHLH1 was most responsive to $\mathrm{NaCl}$ treatment. After $3 \mathrm{~h}$ of $\mathrm{ABA}$ treatment, the expression level of TabHLH1 reached 13- fold and had the same tendency as its expression under $\mathrm{NaCl}$ treatment. The expression level of TabHLH1 was also affected by $100 \mu \mathrm{M}$ MeJA; TabHLH1 expression in leaves increased at $3 \mathrm{~h}$ and then slowly declined to approximately 1.3 -fold at $24 \mathrm{~h}$. Similar results were obtained with SA, ET, and GA3 hormone treatments (Fig. 4E).

\section{Identification of transgenic plants}

To evaluate the regulatory mechanism of TabHLH1 in $T$. antungense polyphenol biosynthesis, the overexpression vector pRI101-TabHLH1-YFP was constructed and transformed into $T$. antungense leaves according to Liu et al. $(2018)^{14}$. Seventeen independent transgenic lines were identified using p35SF as the forward primer (according to the $35 \mathrm{~S}$ promoter sequence) 


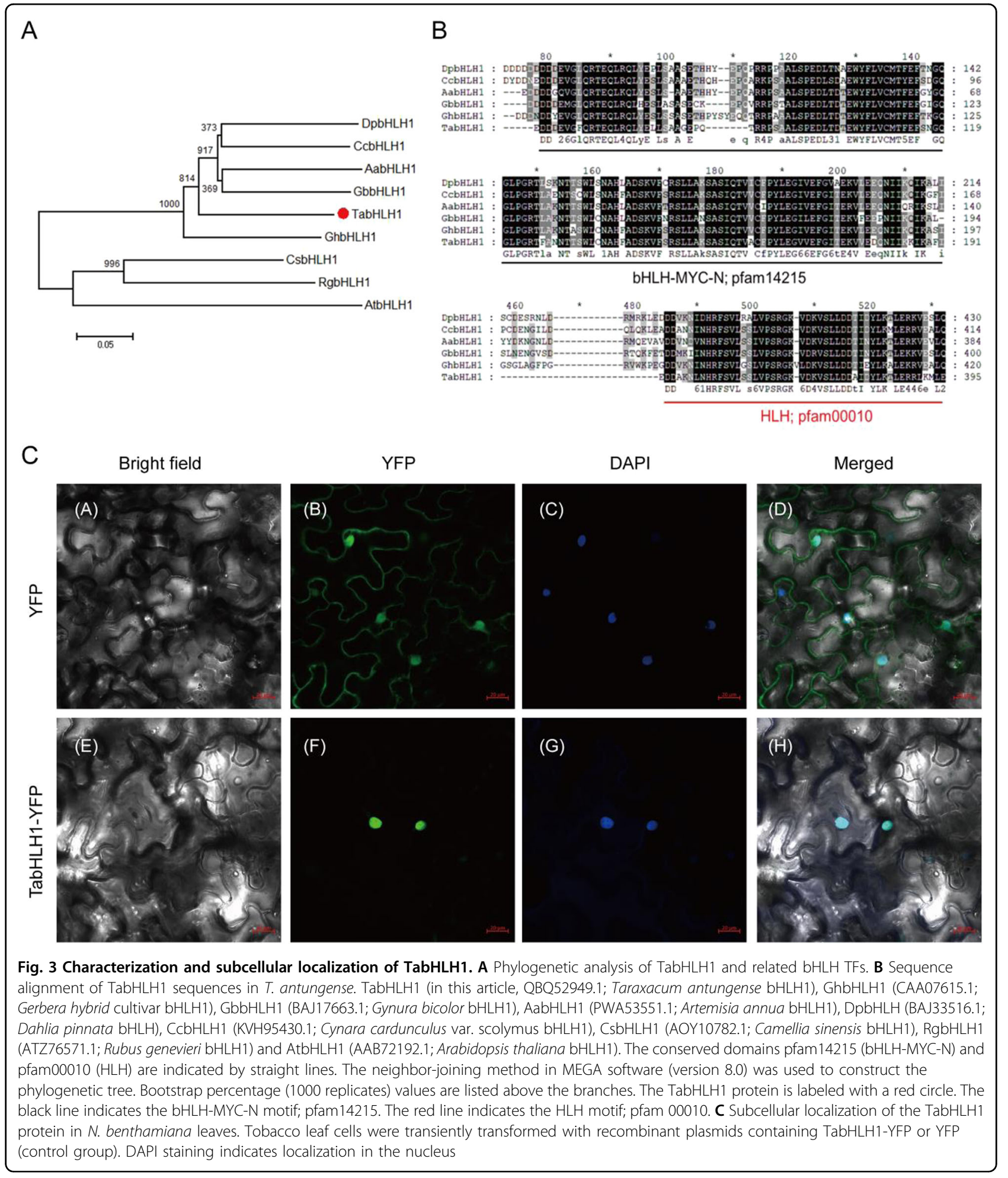

and TabHLH1R as the reverse primer through genomic PCR (Table S1). Three transgenic lines (TabHLH1-OE2, TabHLH1-OE7, and TabHLH1-OE13) with higher expression levels of OE-TabHLH1 than the control lines were selected for further experiments (Fig. S3 and Fig.
5A). At the same time, three RNAi-TabHLH1 transgenic lines (RNAi-1, RNAi-19, and RNAi-22) containing pCAMBIA1300-35S-TabHLH1 were identified according to Liu et al., $2019^{18}$. qRT-PCR showed that TabHLH1 expression levels in the OE-TabHLH1 transgenic lines 
A

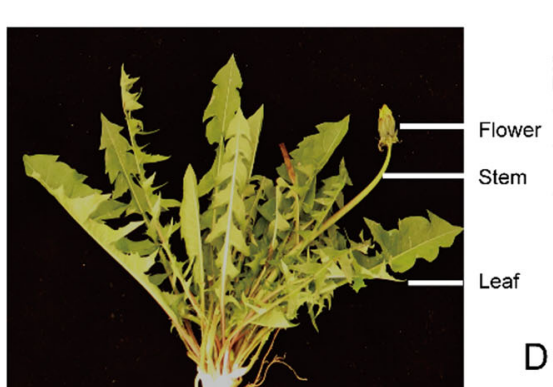

C

D
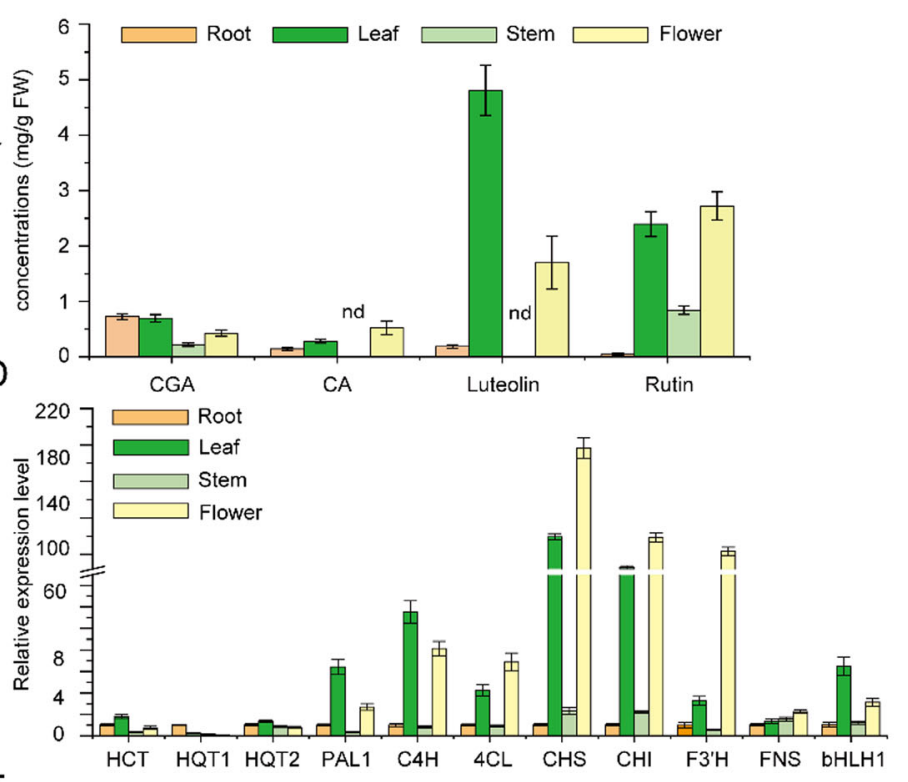

$\mathrm{E}$

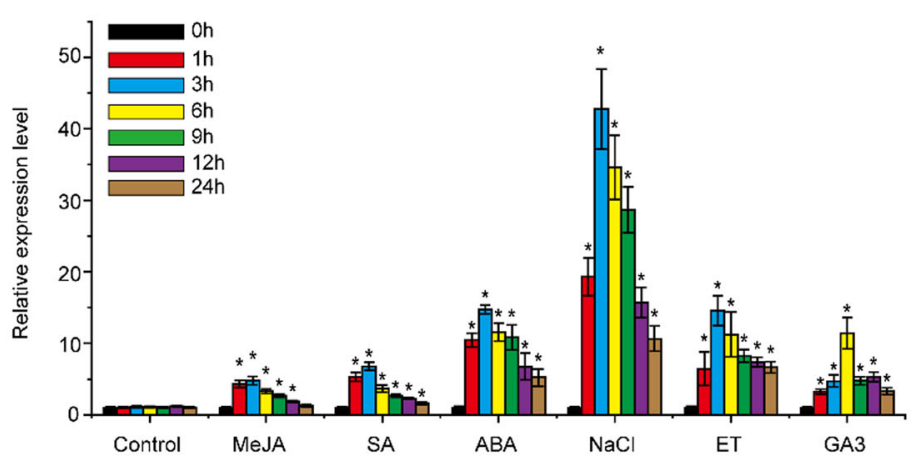

Fig. 4 Basic biochemical analyses of $T$. antungense and the TabHLH1 elicitor response. A Different tissues (leaves, roots, flowers, and stem) of wild T. antungense. B Total polyphenol concentrations in different tissues. C CGA, CA, luteolin, and rutin concentrations in different tissues. nd: not detected. D The expression levels of polyphenol biosynthesis pathway enzyme genes (PAL, 4CL, C4H, HCT, HQT1, HQT2, CHS, CHI, FNS, F3'H) and TabHLH1 TF in different tissues of $T$. antungense. The expression levels of different genes in roots were set to 1 . $\mathbf{E}$ The effects of different hormones on TabHLH1 gene expression levels. Treatment with $100 \mu \mathrm{M}$ methyl jasmonate (MeJA), $100 \mu \mathrm{M}$ salicylic acid (SA), $100 \mu \mathrm{M}$ abscisic acid (ABA), $100 \mu \mathrm{M}$ gibberellin (GA3), $100 \mu \mathrm{M}$ ethylene (ET), or $500 \mu \mathrm{M} \mathrm{NaCl}$ for different time points $(0,1,3,6,9,12,16$, and $24 \mathrm{~h})$. The expression levels of TabHLH1 at $0 \mathrm{~h}$ were set to 1. The TaActin gene was selected as an internal reference in this article

were significantly higher than those in the wild-type (WT) line, while the expression levels of RNAi-TabHLH1 were lower than those in the WT transgenic lines (Fig. 5B).

\section{Role of TabHLH1 in polyphenol biosynthesis}

The concentrations of CGA, CA, rutin, and luteolin in the TabHLH1 transgenic lines were determined by highperformance liquid chromatography (HPLC). CGA concentrations increased in the transgenic lines compared to those in the control lines $(0.73 \pm 0.054 \mathrm{mg} / \mathrm{g}$ dry weight $[\mathrm{DW}])$, with the highest concentration in OE-7 (1.18 \pm $0.113 \mathrm{mg} / \mathrm{g} \mathrm{DW})$ and the lowest in RNAi-19 (0.34 \pm $0.141 \mathrm{mg} / \mathrm{g} \mathrm{DW})$. There were no significant differences in
$\mathrm{CA}$ and rutin concentrations between the transgenic and control lines (Fig. 5C). Luteolin concentrations in OE-13 $(7.07 \pm 0.687 \mathrm{mg} / \mathrm{g} \mathrm{DW})$ increased compared to those in the control lines $(4.83 \pm 0.345 \mathrm{mg} / \mathrm{g} \mathrm{DW})$ and were lowest in RNAi-19 (3.84 $\pm 0.441 \mathrm{mg} / \mathrm{g}$ DW). To identify the CGA and luteolin biosynthetic genes regulated by TabHLH1, the expression levels of key enzyme genes in the CGA and luteolin biosynthesis pathways in $T$. antungense were first determined. Ta4CL, TaHQT2, TaCHI, and $\mathrm{TaF}^{\prime} H$ were all upregulated in the TabHLH1-overexpression lines to various degrees. TaHQT2 expression was the most significantly increased among the four upregulated genes (Fig. 5D). 


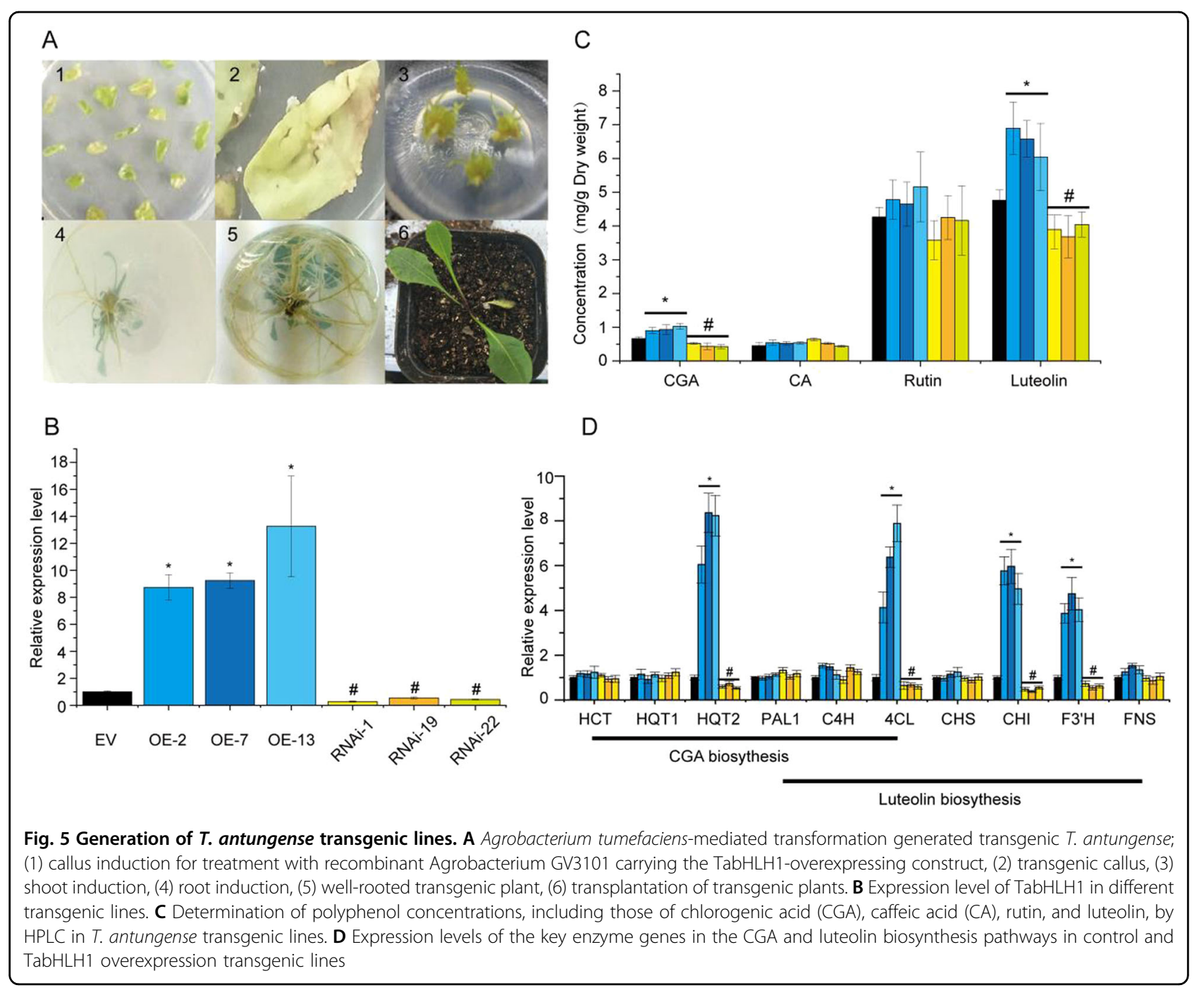

In vivo and in vitro evaluation showed that TabHLH1 increased TaHQT2 and Ta4CL expression

The gene promoters of TaPAL, TaC4H, Ta4CL, TaHCT, and TaHQT1/2 from the CGA biosynthetic pathway all contain bHLH cis-acting elements ${ }^{33}$. Herein, dual-luciferase (LUC) assays were performed to investigate whether TabHLH1 increased the expression of these genes. Reporter and effector vector construction are shown in Fig. 6A. Fluorescence analysis indicated the intensity of gene expression (Fig. 6A). Of the six examined genes, the ratio of LUC/Renilla (REN) was detected only for Ta4CL and TaHQT2 and was significantly higher than that of the control, with a 6.26- and 10.08-fold increase, respectively (Fig. 6B). Furthermore, TabHLH1 binding sites (bHLH-responsive cis-elements) were detected using $\mathrm{Y} 1 \mathrm{H}$ assays and electrophoretic mobility shift assays (EMSAs). TabHLH1 directly combined with the TaHQT2 and Ta4CL promoters through the E-box motif
(CATGTG) (Fig. 6C, D). These results indicated that TabHLH1 directly increased TaHQT2 and Ta4CL gene expression, thereby modulating CGA accumulation.

\section{TabHLH1 increased luteolin biosynthesis}

Luteolin is also produced in plants using phenylalanine as a precursor ${ }^{16}$. Hence, we measured luteolin concentrations in the transgenic lines. Luteolin concentrations were significantly higher in the OE-TabHLH1 transgenic line and lower in the RNAi-TabHLH1 line than in the control groups (Fig. $5 \mathrm{C}$ ). In addition, two of the luteolin biosynthetic pathway genes, $\mathrm{TaCHI}$ and $\mathrm{TaF3}^{\prime} \mathrm{H}$, were strongly upregulated in the OE-TabHLH1 line, while $\mathrm{TaCHI}$ and $\mathrm{TaF3}^{\prime} \mathrm{H}$ were significantly downregulated in the RNAi-TabHLH1 line compared to that in the control groups (Fig. 5D). Dual-LUC assays showed that TabHLH1 significantly increased TaCHI (harboring the CATGTG motif in their promoter) and $\mathrm{TaF3}^{\prime} H$ (without the 

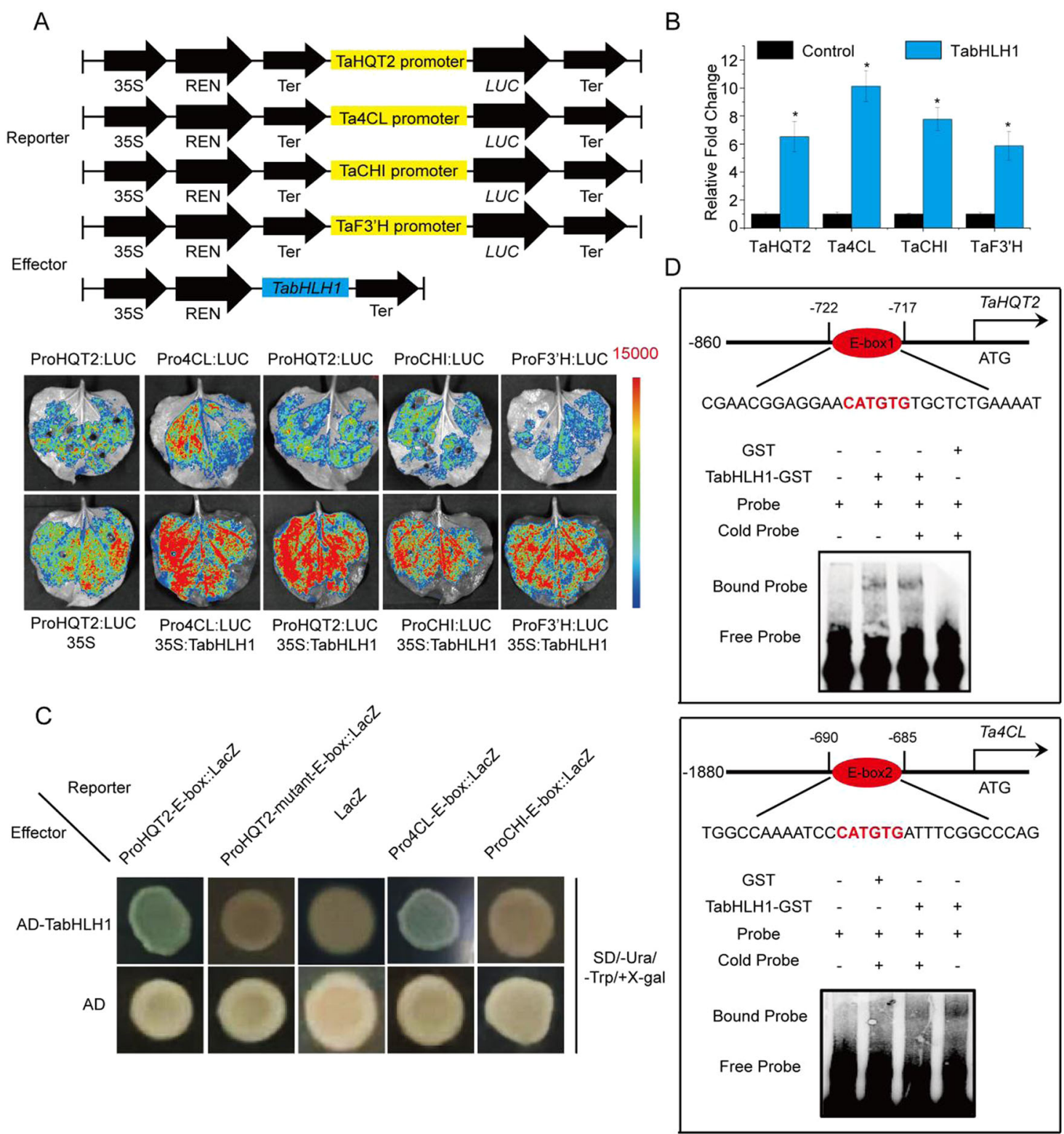

Fig. 6 Dual-LUC and EMSAs proved that TabHLH1 activates the expression of Ta4CL, TaHQT2, TaCHI, and TaF3'H. A Dual-LUC assays indicating that TabHLH1 increases the expression levels of Ta4CL, TaHQT2, TaCHI, and TaF3'H. B The relative fold change of LUC/REN (Renilla) indicates that the expression levels of Ta4CL, TaHQT2, TaCHI, and TaF3'H can be activated by TabHLH1. C Y1H assay results indicated that TabHLH1 directly binds to Ta4CL and TaHQT2 in yeast cells. The CATGTG motif has not been found in the promoter of TaF3'H. D EMSA results indicate that TabHLH1 directly binds to the E-box elements (CATGTG) from the TaHQT2 and Ta4CL promoters. The cold-probe sequences were the same as the labeled probes but without biotin labeling

CATGTG motif) expression levels. The ratio of LUC/REN increased by 7.94-fold for TaCHI and 6.11-fold for TaF3' $H$ compared to that in the control groups (Fig. 6B). However, $\mathrm{Y} 1 \mathrm{H}$ assay results indicated that the E-box (CATGTG motif) in the $\mathrm{TaCHI}$ promoter cannot directly interact with TabHLH1 (Fig. 6C). Together, these results indicated that TabHLH1 increased both CGA and luteolin concentrations in $T$. antungense transgenic lines.

\section{Discussion}

Basic information on the polyphenols in $T$. antungense

In different tissues of $T$. antungense, the total polyphenol concentrations are highest among flowers, followed by leaves, roots, and stems, showing the same tendency as in $T$. mongolicum ${ }^{4}$. Total polyphenols, including chlorogenic, caffeic, quinic, caffeoylquinic, ferulic, cinnamic, caftaric, benzoic, vanillic, protocatechuic, gallic, cumaric acid, 
chrysoeriol, and vanillin, have been found in Taraxacum species $^{1}$. Flowers and leaves have higher polyphenol concentrations than roots and stems. The CGA and CA levels in T. mongolicum were generally lower than those in $T$. antungense, while luteolin levels were significantly higher ${ }^{4}$. The possible reasons are various factors, including growth environments, sample extraction, and HPLC methods, which together cause significant differences in CGA, CA, rutin, and luteolin concentrations in $T$. antungense and closely related species ${ }^{4,21,37}$. The relationship among the four compounds and total polyphenol concentrations should be further studied.

\section{TabHLH1 potentially regulates polyphenols in $T$. antungense}

First, the HQT2 gene promoter was used as bait to screen functional TFs. CGA is widely distributed in many plants, such as T. antungense, L. japonica, Solanum lycopersicum, and Solanum tuberosum ${ }^{20,21,38}$. In the CGA biosynthesis pathway, key enzyme genes, such as PAL, $\mathrm{C} 4 \mathrm{H}, 4 \mathrm{CL}$, and $\mathrm{HCT} / \mathrm{HQT}$, are widely reported ${ }^{14,20}$. However, the regulation of CGA biosynthesis targeting the key enzyme HQT has not been previously reported $^{20,21}$. Gene promoters are the core link between TFs and structural gene expression ${ }^{39-41}$. Through analysis of the TaHQT2 promoter (four CANNTG motifs), it is speculated that TaHQT2 is regulated by bHLH TFs (Fig. $\mathrm{S} 1$ and Table S2) ${ }^{33,41}$. Herein, using proHQT2 as a probe, we first obtained a bHLH TF named TabHLH1 through $\mathrm{Y} 1 \mathrm{H}$ assays.

Second, coexpression analysis can be used to screen and preliminarily identify the correlation between transcription factors and key enzyme gene expression ${ }^{34,40}$. Under ABA treatment, SmbZIP1 and salvianolic acid biosynthesis pathway gene expression levels significantly increased, and coexpression analysis showed a highly linear relationship ${ }^{40}$. By analyzing TabHLH1 together with polyphenol biosynthesis pathway gene expression levels in T. antungense different tissues, it can be concluded that TabHLH1 potentially regulates the expression of key enzyme genes in polyphenol biosynthesis and thus affects the concentration of polyphenols in T. antungense $^{32,33}$. Therefore, polyphenol analysis, $\mathrm{Y} 1 \mathrm{H}$ assays, and coexpression analysis provide a theoretical basis for screening related TFs for designing molecular breeding strategies to improve $T$. antungense quality.

\section{TabHLH1 increased CGA biosynthesis in T. antungense}

In this study, full-length TabHLH1 was isolated and cloned from $T$. antungense and showed the highest identity with the Gerbera hybrid cultivar GhbHLH1. Both GbbHLH1 and GhbHLH1 play a vital role in the regulation of anthocyanin and dihydroflavonol accumulation $^{37,42,43}$. In plants, anthocyanin and CGA have the same precursor and share the first three key enzymes ${ }^{41}$. In this study, salt stress significantly increased TabHLH1 gene expression levels, with the same tendency as that of GhbHLH1, which is consistent with earlier findings ${ }^{42}$. However, under ABA treatment, TabHLH1 expression increased more than 10-fold, which has not been reported in $G b b H L H 1^{42}$. A possible reason for this finding may be species evolution and functional redundancy in multiple gene families ${ }^{31}$. TabHLH1 is located in the nucleus, similar to other nucleus-localized bHLH TFs, such as AaMYC2-Like and MdMYC2 ${ }^{44,45}$. Therefore, TabHLH1 plays a vital role in hormone and salt stress signal regulation, which ultimately assists plants in their response to a variety of biotic and abiotic stresses in their natural environment $^{14,26}$. The relationship among hormones, salt stress signal regulation, and TabHLH1 expression should be further studied.

Multiple TFs, such as SmbHLH37 and SmbHLH53, increase polyphenol concentrations through PAL or other key enzymes ${ }^{32,33}$. By analyzing the CGA concentrations in TabHLH1 transgenic lines (OE-7 and RNAi-19), CGA concentrations in the $\mathrm{OE}-7$ transgenic line increased nearly $63.6 \%$ compared to that in the WT, while CGA concentrations in RNAi-19 decreased to $53.2 \%$, suggesting that TabHLH1 significantly increased CGA concentrations in $T$. antungense. TaHQT2 was identified as the key enzyme that can directly synthesize CGA in previous studies ${ }^{21}$. In this study, Ta4CL and TaHQT2 gene expression levels were positively correlated with the CGA concentration. Furthermore, dual-LUC and EMSA demonstrated that TabHLH1 bound directly to the TaHQT2 promoter region. These results together demonstrated that TaHQT2 was a target of TabHLH1; thus, TabHLH1 played a positive role in regulating CGA biosynthesis.

$\mathrm{p}$-Coumarin-CoA is a precursor compound for the synthesis of downstream phenolic acids, flavonoids, and anthocyanins $^{7,42}$. 4CL participates in the front-end enzymatic reaction of polyphenol biosynthesis and directly catalyzes the biosynthesis of p-coumarin-CoA (Fig. 1). In this study, TabHLH1 increased the expression level of Ta4CL, thereby regulating metabolic flow in plants to accumulate polyphenols, which is consistent with CsbHLH1 directly activating the Cs4CL promoter ${ }^{44,46,47}$. Dual-LUC, Y1H, and EMSA demonstrated that TabHLH1 bound directly to the Ta4CL promoter region. These results suggested that Ta4CL was another target of TabHLH1.

TabHLH1 increased $\mathrm{CHI}$ and $\mathrm{F}^{\prime} \mathrm{H}$ gene expression levels in vivo to promote luteolin biosynthesis in $T$. antungense

Flavonoid biosynthesis regulation has been studied indepth, particularly in the medicinal plant $S$. miltiorrhiza ${ }^{19,33,48}$. Functional genes, including key enzyme genes and numerous TFs, are required for luteolin 
biosynthesis $^{29,44}$. In this study, TabHLH1 significantly increased luteolin concentrations (OE-7 increased to 1.46-fold, and RNAi-19 decreased to 0.78-fold) in transgenic lines compared to that in the WT. In addition, concentrations of the key luteolin biosynthesis enzymes $\mathrm{TaCHI}$ and $\mathrm{TaF3}^{\prime} \mathrm{H}$ were directly influenced by overexpression/RNAi of TabHLH1, suggesting that TabHLH1 may interact with $T a C H I$ and $T a F 3^{\prime} H$. Dual-LUC results showed that TabHLH1 increased the expression levels of two pathway genes involved in luteolin biosynthesis. However, TabHLH1 binds indirectly to the promoters of $\mathrm{TaCHI}$ and $\mathrm{TaF}^{\prime} \mathrm{H}$ (Fig. 6C). The possible reason may be that TabHLH1 interacts with other proteins, such as MYB partners, which can directly interact with $\mathrm{TaCHI}$ and $\mathrm{TaF3}^{\prime} H$. These results indicated that TabHLH1 was positively involved in regulating luteolin biosynthesis and metabolic flow, and the direct target was Ta4CL, while the potential targets were $\mathrm{TaCHI}$ and $\mathrm{TaF}^{\prime} \mathrm{H}$. The MYBbHLH-WD40 complex often increases the expression level of flavonoid biosynthesis genes to control the production of anthocyanins ${ }^{49}$. CsbHLH1 (CsMYC1) interacts with CsbHLH42, CsWD4O, CsMYB60 and itself to regulate flavonoid biosynthesis in cucumber ${ }^{47}$. In T. antungense, further studies should focus on proteins that interact with TabHLH1 to regulate polyphenol biosynthesis.

Based on these results, a functional model for the role of TabHLH1 in polyphenol biosynthetic regulation in $T$. antungense is provided (Fig. 7). In summary, TabHLH1 increased TaHQT2 and Ta4CL expression levels, leading to increased CGA concentrations. This is the first dandelion bHLH protein identified as being involved in CGA pathway regulation. In addition, TabHLH1 promoted the expression of luteolin biosynthesis genes ( $\mathrm{TaCHI}$ and $\mathrm{TaF}^{\prime} H$ ) to increase the accumulation of luteolin. These studies provide new insights into the role of TabHLH1 in the regulation of polyphenol biosynthesis. In addition, these findings lay the foundation for further exploration of the molecular mechanisms and potential functional genes of secondary metabolite biosynthesis in T. antungense.

\section{Materials and Methods \\ Plant materials}

T. antungense biomaterials were collected and transplanted in the greenhouse of our laboratory, as previously reported $^{34} . N$. benthamiana and T. antungense seeds were sown in substrate/vermiculite (3/1)-admixture soil and transplanted in pots for 4-6 weeks for hormone treatment experiments. Plants were maintained at a constant temperature of $25^{\circ} \mathrm{C}$ under $16 / 8 \mathrm{~h}$ light/dark cycles for use in transient expression analysis ${ }^{14}$.

\section{TaHQT2 promoter cloning and $\mathrm{Y} 1 \mathrm{H}$ screening}

The CTAB method was used to extract dandelion plant DNA, and RNase was used to remove the remaining RNA.

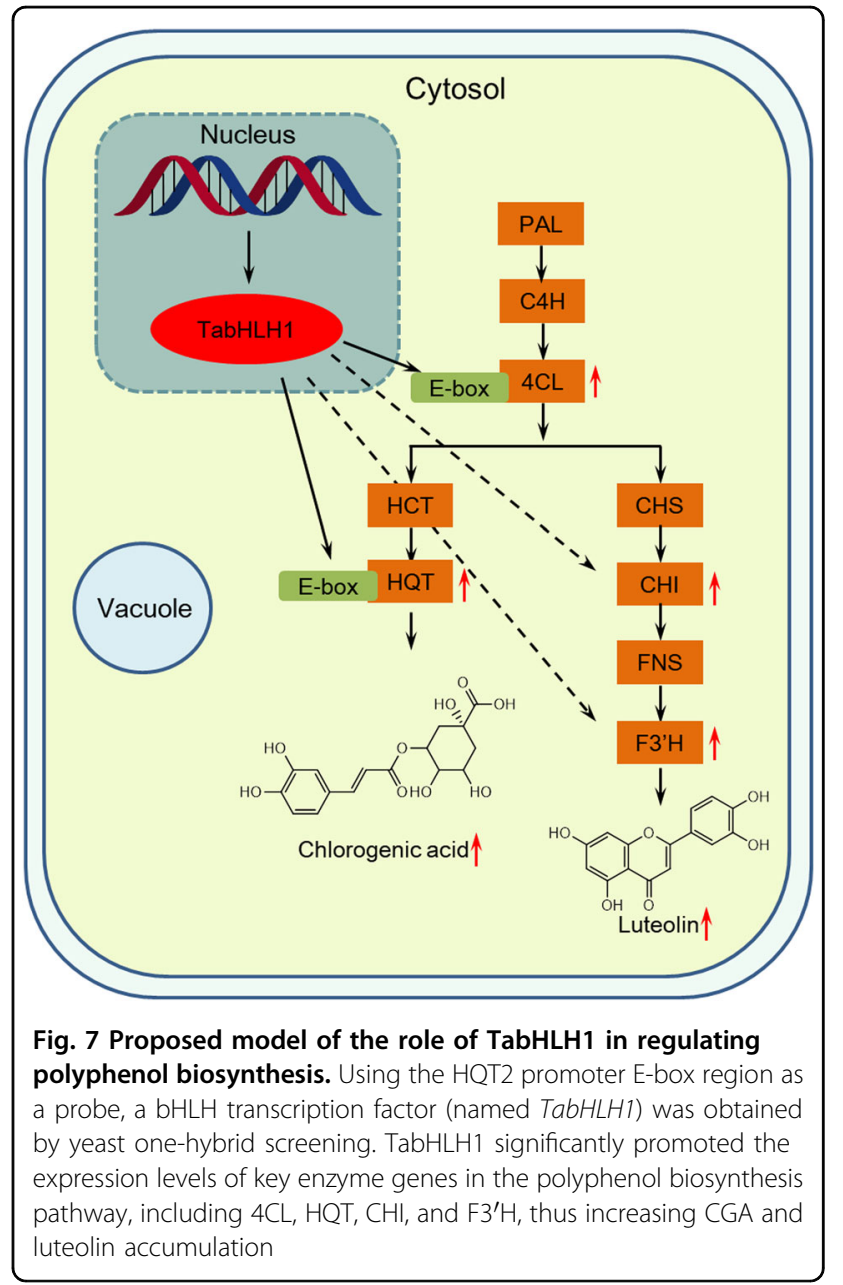

Fusion primers and nested integrated PCR were used to obtain the TaHQT2 $5^{\prime}$-end promoter ${ }^{47,50}$. All primers are listed in Table S1. PlantCARE (http://bioinformatics.psb. ugent.be/webtools/plantcare/html/) was used to analyze the promoter cis-element sequences.

The TaHQT2 promoter (four E-boxes located from -691 bp to -806 bp, Fig. S1) was constructed in pABAi to create the pAbAi-proTaHQT2 recombinant vector using the BamH I and Hind III restriction sites. BstBI was used to digest recombinant plasmids, linearized pAbAiproTaHQT2 plasmids were transformed into the yeast strain $(\mathrm{Y} 1 \mathrm{H})$, and then, the resulting strains were tested on $\mathrm{SD}$ /-Ura media containing aureobasidin $\mathrm{A}(\mathrm{AbA})$ at concentrations ranging from $100-500 \mathrm{ng} / \mathrm{mL}$.

A Y1H cDNA library of $T$. antungense was constructed using the Matchmaker ${ }^{\mathrm{TM}}$ one-hybrid library construction \& screening kit PT3529-1 (PR732190, Takara Biomedical Technology Co., Ltd., Beijing, China). Yeast recombinant vector (GAL4-AD-sec) was extracted from the primary library and transformed into $\mathrm{Y} 1 \mathrm{H}$ containing $\mathrm{pAbAi}$ proTaHQT2. After rescreening on SD/-Leu-Ura medium with higher $\mathrm{AbA}$ concentrations than listed above, 
positive pGADT7-sequence yeast strains were used for sequencing analysis. The PCR thermocycler program used was as follows: $94^{\circ} \mathrm{C}$ for $10 \mathrm{~min}, 40$ cycles of denaturation, annealing, and extension $\left(94^{\circ} \mathrm{C}\right.$ for $30 \mathrm{~s}, 50^{\circ} \mathrm{C}$ for $45 \mathrm{~s}$, and $72{ }^{\circ} \mathrm{C}$ for $90 \mathrm{~s}$, respectively), and a final extension at $72^{\circ} \mathrm{C}$ for $10 \mathrm{~min}$.

\section{Bioinformatics analysis and isolation of TabHLH1}

A bHLH TF protein isolated from $T$. antungense, which was designated TabHLH1, was found to interact with the E-boxes of TaHQT2. The gene sequence was compared to Taraxacum kok-saghyz Rodin (accession number: GWHAAAAM043215) ${ }^{36}$. The complete coding sequence was obtained using homologous cloning. The target cDNA fragment was connected to the $18 \mathrm{~T}$ vector (Takara Biomedical Technology Co., Ltd., Beijing, China) for sequencing analysis. BLAST alignment (http://www.ncbi. nlm.nih.gov/BLAST/) was used to search for orthologs. ClustalX (version 1.81) was used for multiple sequence alignment through the neighbor-joining method using 1000 repetitions. A molecular phylogenetic tree was constructed using the MEGA program (version 8.0) ${ }^{14}$.

\section{Elicitor treatment and subcellular localization of TabHLH1}

Methyl jasmonate (MeJA), salicylic acid (SA), ethephon (ET), gibberellin (GA), and abscisic acid (ABA) purchased from Sigma-Aldrich (Shanghai, China) were directly dissolved in distilled water at a final concentration of $100 \mathrm{mM}$. NaCl (Aladdin, Shanghai, China) was dissolved at a concentration of $500 \mathrm{mM}$, and distilled water was used as a control. Two-month-old well-grown T. antungense transgenic and WT plants were chosen for different treatments. The above elicitors were filter-sterilized through a $0.45 \mu \mathrm{m}$ filter membrane (Pall Corporation, NY, USA) and added to the cultures at a final concentration of $100 \mu \mathrm{M}$. Tissues from different parts of the plants were collected after $0,1,3,6,9,12,16$, and $24 \mathrm{~h}$ of treatment.

To identify the in vivo subcellular location of TabHLH1, the coding sequence of TabHLH1 was fused with a reporter sequence. The complete coding sequence of TabHLH1 (without the TAG stop codon), including the restriction sites $\mathrm{Nde}$ I (in the $5^{\prime}$-end) and $\mathrm{Sal}$ I (in the 3'end), was amplified and subcloned into the pMD19-T simple vector (Takara Biomedical Technology Co., Ltd., Beijing, China). The plasmid pRI101-YFP (containing the yellow fluorescent protein gene) was double-digested using the same enzymes to create a recombinant vector termed pRI101-TabHLH1-YFP, and the insert was sequenced using the 35S (forward) and TabHLH1R (reverse) primers (Fig. S2 and Table S1). The fused recombinant expression plasmid was transformed into $N$. tabacum. pRI101-YFP was used as a control to perform the transient expression assay ${ }^{21,40}$.

\section{Transformation of $T$. antungense}

The pCAMBIA1300-35S-X (restriction sites: BamH I/ Spe I for sense and Kpn I/Sac I for antisense sequences) RNAi expression vector was used for RNAi-TabHLH1 recombinant plasmid creation ${ }^{21}$. The SPLRNAi gene was used as intron $\mathrm{X}$. The middle region of TabHLH1 (631-839 bp) was used for vector construction (nonconserved region). Both pCAMBIA1300-35S-TabHLH1 and pRI101-TabHLH1-YFP were transformed into Agrobacterium tumefaciens strain GV3101. pCAMBIA1300$35 \mathrm{~S}-\mathrm{X}$ and the pRI101-YFP vector were used as controls. After positive identification, Agrobacterium harboring different recombinant plasmids were used for injection into plants. Following a previously published protocol ${ }^{14}$, Agrobacterium infection was used for genetic transformation to obtain $T$. antungense transgenic plants.

\section{Analysis of gene expression profiles}

Different tissues or transgenic lines of $T$. antungense were used for total RNA extraction, followed by cDNA synthesis, which was performed following the abovementioned methods ${ }^{50,51}$. qRT-PCR was performed using gene-specific primer pairs for $P A L, C 4 H, 4 C L, H C T$, $H Q T 2, C H S, C H I$, and $F 3^{\prime} H$ (Supplemental Table S1) using three technical replicates. Based on the $2^{-\Delta \Delta \mathrm{Ct}}$ method, qRT-PCR was performed, and relative expression levels were calculated using $\beta$-actin as a reference gene ${ }^{14}$.

\section{Measurement of polyphenol concentrations by HPLC}

HPLC was used to investigate the concentrations of four polyphenols (CGA, CA, rutin, and luteolin) in the $T$. antungense plant materials from the control groups and transgenic lines (Table S3). For transgenic lines, 3-monthold whole plants (containing roots and leaves) were dried and dehydrated at $-20^{\circ} \mathrm{C}$ to constant weight, ground into powder, and then used as samples. Samples were ultrasonically extracted for polyphenol compounds and passed through a $0.22 \mu \mathrm{m}$ filter membrane for HPLC, as previously described ${ }^{14,23}$. HPLC conditions for polyphenol detection were as described in the previous reports ${ }^{34}$.

\section{Measurement of total phenolic concentrations}

The total phenolic concentrations of T. antungense in different tissues were extracted with Folin-Ciocalteu reagent as previously reported ${ }^{4,5}$. Then, $500 \mu \mathrm{L}$ of $T$. antungense extract was added to $1.5 \mathrm{~mL}$ FC reagent $(0.2 \mathrm{mg} / \mathrm{mL})$ and mixed. Two milliliters of $7.5 \% \mathrm{Na}_{2} \mathrm{CO}_{3}$ reagent and $2 \mathrm{~mL}$ distilled water were added. Then, the mixture was incubated at $25^{\circ} \mathrm{C}$ for $1 \mathrm{~h}$ (in the dark). The absorbance of the mixture was recorded at $727 \mathrm{~nm}$, and $60 \%$ methanol was used as a control. The total phenolic concentrations of $T$. antungense samples were calculated according to milligrams of gallic acid equivalents per gram dry weight of the sample (mg GAE/g) 


\section{Dual-LUC assay}

The pCAMBIA2300 ${ }^{+}$-TabHLH1 vector acted as an effector and was transferred into A. tumefaciens strain GV3101 (pCAMBIA2300 ${ }^{+}$-vector was used as a control). The promoters of key enzyme genes, including Ta4CL, $T a H Q T 2$, $T a C H I$, and $T a F 3^{\prime} H$, were cloned separately into the pGreen0800-rec plasmid. The pGreen0800promoter recombinant vectors, separately with the helper vector pSoup19, were cotransformed into GV3101. The Renilla vector was used as an internal control. Both the reporter and effector strains were mixed in equal proportions ( $3 \mathrm{~mL}$ each), slowly cultivated for $2 \mathrm{~h}$, and injected into the leaves of 2-month-old $N$. benthamiana. After incubation in the dark for 2-3 days, commercial dual-LUC reaction reagents (Promega Biomedical Technology Co., Ltd., Beijing, China) were used to perform dual-LUC assays on leaf samples ${ }^{18}$. Three biological replicates were measured for each sample.

\section{$\mathrm{Y} 1 \mathrm{H}$ assay}

The $\mathrm{Y} 1 \mathrm{H}$ assay was performed differently from the $\mathrm{Y} 1 \mathrm{H}$ screening and used the $\mathrm{pB} 42 \mathrm{AD} / \mathrm{pLacZ}$ system, which has been previously described ${ }^{40}$. The full-length TabHLH1 ORF fragment was amplified, sequenced, and cloned into the effector plasmid (pB42AD). For the reporter plasmid pLacZ, a triple tandem copy of the E-box (NNNNCATGTGNNNN) motif near $4 \mathrm{bp}$ from every promoter (TaHQT2, Ta4CL, TaCHI) was inserted by using EcoR I and Xho I as restriction endonuclease sites. After identification by sequencing, recombinant effector plasmids (pB42AD-TabHLH1) and recombinant reporter plasmids (pLacZ-TaHQT2, pLacZ-Ta4CL, pLacZ-TaCHI) were cotransformed into yeast strain EGY48a. Transformants were cultivated on SD/-Ura/-Trp medium for $2 \mathrm{~d}$ and then transferred (by using distilled water-diluted 1000 -fold) to SD/-Ura/-Trp medium with 5-bromo-4chloro-3-indolyl- $\beta$-D-galactopyranoside (X-gal) for another 1-2 d. Empty pB42AD and pLacZ plasmids were used as negative controls and cotransformed into EGY48a strains.

\section{EMSAs}

The complete sequence of TabHLH1 was inserted into the BamH I and Sal I sites of the pGEX4T-1 plasmid and then transformed into Escherichia coli (BL21 or DE3 strain). Isopropyl-D-thiogalactoside was used to induce recombinant protein expression overnight $(16 \mathrm{~h})$, and the GSTtagged protein purification kit (Transgen Biotech Co., Ltd., Beijing, China) was used to purify recombinant proteins. Biotin-labeled 5'- and 3'-ends of the TaHQT2 promoter were synthesized by Shanghai Sangon Co. (Shanghai, China), and the two biotin-labeled primers were annealed to form double-stranded DNA fragments. The purified recombinant proteins and DNA fragments were incubated in 10× EMSA binding buffer (Beyotime Biotechnology Co., Ltd., Shanghai, China) at $25^{\circ} \mathrm{C}$ for $30 \mathrm{~min}$. DNA fragments without biotin labeling were used as an internal control. The DNA-protein complex was electrotransferred to a wet electromembrane and examined following the manufacturer's instructions by using a chemiluminescent nucleic acid detection module kit (Beyotime Biotechnology Co., Ltd., Shanghai, China) ${ }^{19,27,48,52}$.

\section{Statistical analyses}

Statistical comparisons were performed using SPSS v19.0 software. Error bars represent the SE of three biological replicates. All data are presented as the mean \pm standard deviation (SD). Statistical significance was assessed using Student's $t$ test (\#: decrease, ": increase, $P<$ 0.05 ) for all the experiments involved in this article (for the different tissues, transgenic lines, and control groups).

\section{Acknowledgements}

This work was supported by the Zhejiang Provincial Ten Thousand Program for Leading Talents of Science and Technology Innovation [2018R52050], the National Natural Science Fund of China [Grant numbers: 82073963, 81522049, 81703636, 31571735, 31270007], the Zhejiang Provincial Program for the Cultivation of High-Level Innovative Health Talents, the Research Project of Zhejiang Chinese Medical University (2021JKZDZC06), the Opening Project of Zhejiang Provincial Preponderant and Characteristic Subject of Key University (Traditional Chinese Pharmacology), Zhejiang Chinese Medical University [ZYAOXZD2019006]. Funds were used for the collection and analysis of data in this study, as well as for the open access payment. We appreciate the experimental support from the Public Platform of Medical Research Center, Academy of Chinese Medical Science, Zhejiang Chinese Medical University.

\section{Author details}

${ }^{1}$ Laboratory for Core Technology of TCM Quality Improvement and Transformation, College of Pharmacy, School of Pharmaceutical Sciences, The Third Affiliated Hospital, Zhejiang Chinese Medical University, Hangzhou, Zhejiang 310053, PR China. ${ }^{2}$ College of Horticulture, Shenyang Agricultural University, Shenyang 110866, China. ${ }^{3}$ Institute of Botany, Jiangsu Province and Chinese Academy of Sciences (Nanjing Botanical Garden Mem.Sun Yat-Sen), Nanjing 210014, China. ${ }^{4}$ College of Traditional Chinese Materia Medica, Shenyang Pharmaceutical University, Shenyang 110016, PR China. ${ }^{5}$ Guangxi Botanical Garden of Medicinal Plants, Nanning 530023, PR China. ${ }^{6}$ College of Life Sciences and Engineering, Shenyang University, Shenyang 110044, PR China

\section{Author contributions}

L.Q. designed and planned the experiments. L.Q., Y.L., W. J, C.H., and H.H. collected the materials. L.Q., L. L, and H.H. performed the experiments. L.Q., L. L, and C.H. compiled and interpreted the data. L.Q. wrote the paper. Prof. K.G. and N. W revised the paper.

Conflict of interest

The authors declare no competing interests.

Supplementary information The online version contains supplementary material available at https://doi.org/10.1038/s41438-021-00630-y.

Received: 9 December 2020 Revised: 30 April 2021 Accepted: 7 June 2021 Published online: 01 September 2021 


\section{References}

1. Martinez, M. et al. Taraxacum officinale and related species-an ethnopharmacological review and its potential as a commercial medicinal plant. J. Ethnopharmacol. 169, 244-262 (2015).

2. Flores-Ocelotl, M. R. et al. Taraxacum officinale and Urtica dioica extracts inhibit dengue virus serotype 2 replication in vitro. BMC Complem Alter. M. 18, 95 (2018).

3. Lis, B. \& Olas, B. Pro-health activity of dandelion (Taraxacum officinale L.) and its food products-history and present. J. Funct. Foods 59, 40-48 (2019).

4. Duan L., Zhang C., Zhao Y., Chang Y., Guo L. Comparison of bioactive phenolic compounds and antioxidant activities of different parts of Taraxacum mongolicum, Molecules 25, 3260 (2020).

5. Li, L. et al. Expression of putative luteolin biosynthesis genes and WRKY transcription factors in Taraxacum antungense kitag. Plant Cell Tiss Org. (2021). https://doi.org/10.1007/s11240-021-02035-0.

6. Han, J. Y. et al. Cloning and characterization of oxidosqualene cyclases involved in Taraxasterol, Taraxerol and Bauerenol triterpene biosynthesis in Taraxacum coreanum. Plant Cell Physiol. 60, 1595-1603 (2019).

7. Zhou, S. et al. Integrative analysis of metabolome and transcriptome reveals anthocyanins biosynthesis regulation in grass species Pennisetum purpureum. Ind. Crop Prod. 138, 1-12 (2019).

8. Liu, C. et al. Metabolic and transcriptomic analysis related to flavonoid biosynthesis during the color formation of Michelia crassipes tepal. Plant Physiol. Bioch. 155, 938-951 (2020).

9. Dong, J. et al. Synthesis and structure-activity relationship studies of a-naphthoflavone derivatives as CYP1B1 inhibitors. Eur. J. Med Chem. 187, 111938 (2020).

10. Qian, J. \& Kai, G. Application of micro/nanomaterials in adsorption and sensing of active ingredients in traditional Chinese medicine. J. Pharm. Biomed. 190 113548 (2020)

11. Colombo, R. \& Papetti, A. An outlook on the role of decaffeinated coffee in neurodegenerative diseases. Crit. Rev. Food Sci. 60, 760-779 (2020).

12. Blanco, E. et al. Isolation and characterization of the flavonol regulator CCMYB12 from the globe Artichoke Cynara cardunculus var. scolymus (L.) Fiori. Front Plant Sci. 9, 941 (2018)

13. Jędrejek, D. et al. Evaluation of antioxidant activity of phenolic fractions from the leavesand petals of dandelion in human plasma treated with $\mathrm{H}_{2} \mathrm{O}_{2}$ and $\mathrm{H}_{2} \mathrm{O}_{2}$ /Fe. Chem. Biol. Interact. 262, 29-37 (2017).

14. Liu, Q. et al. Overexpression of and RNA interference with hydroxycinnamoylCoA quinate hydroxycinnamoyl transferase affect the chlorogenic acid metabolic pathway and enhance salt tolerance in Taraxacum antungense Kitag. Phytochem Let. 28, 116-123 (2018).

15. Hichri, I. et al. The basic helix-loop-helix transcription factor MYC1 is involved in the regulation of the flavonoid biosynthesis pathway in grapevine. Mol. Plant. 3, 509-523 (2010)

16. Dong, J. et al. Discovery of heterocycle-containing a-naphthoflavone derivatives as water-soluble, highly potent and selective CYP1B1 inhibitors. Eur. J. Med Chem. 209, 112895 (2020).

17. Chen, Z. et al. Transcriptome analysis reveals the mechanism underlying the production of a high quantity of chlorogenic acid in young leaves of Lonicera macranthoides Hand.-Mazz. PLoS One 10, e0137212 (2015).

18. Hao, X. et al. Tanshinone and salvianolic acid biosynthesis are regulated by SmMYB98 in Salvia miltiorrhiza hairy roots. J. Adv. Res. 23, 1-12 (2020).

19. Huang, Q. et al. The AP2/ERF transcription factor SmERF1L1 regulates the biosynthesis of tanshinones and phenolic acids in Salvia miltiorrhiza. Food Chem. 274, 368-375 (2019).

20. Moglia, A. et al. Genome-wide identification of BAHD acyltransferases and in vivo characterization of HQT-like enzymes involved in caffeoylquinic acid synthesis in globe artichoke. Front Plant Sci. 7, 1-10 (2016).

21. Liu, Q. et al. In vitro evaluation of hydroxycinnamoyl CoA: quinate hydroxycinnamoyl transferase expression and regulation in Taraxacum antungense in relation to 5-caffeoylquinic acid production. Phytochem 162, 148-156 (2019).

22. Xie, L. et al. Involvement of MdUGT75B1 and MdUGT71B1 in flavonol galactoside/glucoside biosynthesis in apple fruit. Food Chem. 312, 1-11 (2020).

23. Yuan, Y. et al. Exploiting genes and functional diversity of chlorogenic acid and luteolin biosyntheses in Lonicera japonica and their substitutes. Gene 534, 408-416 (2014)

24. Yang, L. L. et al. Drought stress induces biosynthesis of flavonoids in leaves and saikosaponins in roots of Bupleurum chinense DC. Phytochem 177, 1-12 (2020).
25. Yin, Q. et al. Genome-wide analyses reveals a glucosyltransferase involved in rutin and emodin glucoside biosynthesis in tartary buckwheat. Food Chem. 318, 1-11 (2020).

26. Sun, Z. et al. Exogenous application of salicylic acid enhanced the rutin accumulation and influenced the expression patterns of rutin biosynthesis related genes in Fagopyrum tartaricum Gaertn leaves. Plant Growth Regul. 68, 9-15 (2012).

27. Deng, C. et al. Tanshinone production could be increased by the expression of SmWRKY2 in Salvia miltiorrhiza hairy roots. Plant Sci. 284, 1-8 (2019).

28. Shi, M. et al. Simultaneous promotion of tanshinone and phenolic acid biosynthesis in Salvia miltiorrhiza hairy roots by overexpressing Arabidopsis MYC2. Ind. Crop Prod. 155, 1-6 (2020).

29. Zhang, H. B. et al. Tobacco transcription factors NtMYC2a and NtMYC2b form nuclear complexes with the NtJZ1 repressor and regulate multiple jasmonate-inducible steps in nicotine biosynthesis. Mol. Plant. 5, 73-84 (2012).

30. Zhang, $\mathrm{H}$. et al. The basic helix-loop-helix transcription factor CrMYC2 controls the jasmonate-responsive expression of the ORCA genes that regulate alkaloid biosynthesis in Catharanthus roseus. Plant J. 67, 61-71 (2011).

31. Zhou, W. et al. Molecular cloning and characterization of two 1-deoxy-Dxylulose-5-phosphate synthase genes involved in tanshinone biosynthesis in Salvia miltiorrhiza. Mol. Breed. 36, 124 (2016).

32. Hao, X. et al. Effects of methyl jasmonate and salicylic acid on tanshinone production and biosynthetic gene expression in transgenic Salvia miltiorrhiza hairy roots. Biotechnol. Appl Bioc. 62, 24-31 (2015).

33. Peng, J. J. et al. SmbHLH53 is relevant to jasmonate signaling and plays dual roles in regulating the genes for enzymes in the pathway for salvianolic acid B biosynthesis in Salvia miltiorrhiza. Gene 756, 1-10 (2020).

34. Liu, Q. et al. Overexpression of TaWRKY14 transcription factor enhances accumulation of chlorogenic acid in Taraxacum antungense Kitag and increases its resistance to powdery mildew. Plant Cell Tiss. Org. 143, 665-679 (2020).

35. Breeze, E. et al. Master MYCs: MYC2, the jasmonate signaling "master switch". Plant Cell. 31, 9-10 (2019).

36. Lin, T. et al. Genome analysis of Taraxacum kok-saghyz Rodin provides new insights into rubber biosynthesis. Nati Sci. Rev. 5, 78-87 (2018).

37. Elomaa, P. et al. A bHLH transcription factor mediates organ, region and flower type specific signals on dihydroflavonol-4-reductase (dfr) gene expression in the inflorescence of Gerbera hybrida (Asteraceae). P J. 16, 93-99 (2010).

38. Clé, C. et al. Modulation of chlorogenic acid biosynthesis in Solanum lycopersicum; consequences for phenolic accumulation and UV-tolerance. Phytochem 69, 2149-2156 (2008).

39. Shi, M. et al. Biotechnological exploration of transformed root culture for value-added products. Trends in Botechnol. https://doi.org/10.1016/j. tibtech.2020.06.012 (2020).

40. Deng, C. et al. ABA-responsive transcription factor bZIP1 is involved in modulating biosynthesis of phenolic acids and tanshinones in Salvia miltiorrhiza. J. Exp. Bot. 71, 5948-5962 (2020).

41. Shen, Q. et al. The jasmonate-responsive AaMYC2 transcription factor positively regulates artemisinin biosynthesis in Artemisia annua. N. Phytol. 210, 1269-1281 (2016).

42. Shimomura, Y. et al. Co-expression of GbMYB1 and GbMYC1 induces anthocyanin accumulation in roots of cultured Gynura bicolor DC. plantlet on methyl jasmonate treatment. Plant Physiol. Bioch 49, 159-167 (2011).

43. Liu, J., Osbourn, A. \& Ma, P. MYB transcription factors as regulators of phenylpropanoid metabolism in plants. Mol. Plant. 8, 689-708 (2015).

44. Luo, J. et al. AtMYB12 regulates caffeoyl quinic acid and flavonol synthesis in tomato: expression in fruit results in very high levels of both types of polyphenol. P J. 56, 316-326 (2010).

45. Majid, I., Kumar, A. \& Abbas, N. A basic helix loop helix transcription factor, AaMYC2-Like positively regulates artemisinin biosynthesis in Artemisia annua $\mathrm{L}$. Ind. Crop Prod. 128, 115-125 (2019).

46. Li, J. et al. CsMYB60 directly and indirectly activates structural genes to promote the biosynthesis of flavonols and proanthocyanidins in cucumber Hortic. Res. 7, 1-15 (2020).

47. Pandey, A. et al. Expression of Arabidopsis MYB transcription factor, AtMYB111, in tobacco requires light to modulate flavonol content. Sci. Re. 4, 5018 (2014).

48. Sun, M. et al. The biosynthesis of phenolic acids is positively regulated by the JA-responsive transcription factor ERF115 in Salvia miltiorrhiza. J. Exp. Bot. 70, 243-254 (2019)

49. $\mathrm{Xu}, \mathrm{W}$. et al. Complexity and robustness of the flavonoid transcriptional regulatory network revealed by comprehensive analyses of MYB-bHLH-WDR 
complexes and their targets in Arabidopsis seed. N. Phytol. 202, 132-144 (2013).

50. Wang, Z. et al. Fusion primer and nested integrated PCR (FPNI-PCR): a new high-efficiency strategy for rapid chromosome walking or flanking sequence cloning. BMC Biotech 11, 109 (2011).
51. Kai, G. et al. Metabolic engineering tanshinone biosynthetic pathway in Salvia miltiorrhiza hairy root cultures. Metab. Eng. 13, 319-327 (2011).

52. Li, T. et al. Apple (Malus domestica) MdERF2 negatively affects ethylene biosynthesis during fruit ripening by suppressing MdACS1 transcription. P. J $\mathbf{8 8}$ 735-748 (2016). 